\title{
Mutants of Escherichia coli producing pyrroloquinoline quinone
}

\author{
Francis Biville, Evelyne Turlin and Francis Gasser*
}

Unité de Régulation de l'Expression Génétique, Département de Biochimie et Génétique Moléculaire, Institut Pasteur, 28 rue du Dr Roux, F-75724 Paris Cedex 15, France

(Received 30 January 1991; revised 18 April 1991; accepted 2 May 1991)

\begin{abstract}
In glucose minimal medium a PTS ${ }^{-}$strain of Escherichia coli $[\Delta(p t s H$ ptsI crr)] could grow slowly (doubling time, $d=10 \mathrm{~h}$ ). When the population reached $5 \times 10^{6}$ to $2 \times 10^{7}$ cells ml ${ }^{-1}$, mutants growing rapidly $(d=1.5 \mathrm{~h})$ appeared and rapidly outgrew the initial population. These mutants (EF mutants) do not use a constitutive galactose permease for glucose translocation. They synthesize sufficient pyrroloquinoline quinone (PQQ) to yield a specific activity of glucose dehydrogenase (GDH) equivalent to that found in the parent strain grown in glucose minimal medium supplemented with $1 \mathrm{nM}-\mathrm{PQQ}$. Membrane preparations containing an active GDH oxidized glucose to gluconic acid, which was also present in the culture supernatant of EF strains in glucose minimal medium. Glucose utilization is the only phenotypic trait distinguishing EF mutants from the parent strain. Glucose utilization by EF mutants was strictly aerobic as expected from a PQQ-dependent catabolism. The regulation of PQQ production by $E$. coli is discussed.
\end{abstract}

\section{Introduction}

Pyrroloquinoline quinone (PQQ) is now recognized as the third important redox cofactor in bacterial dehydrogenases beside NAD(P) and flavins (Duine \& Jongejan, 1990). In Gram-negative bacteria, several membrane or periplasmic dehydrogenases used for the primary oxidation step of non-phosphorylated substrates are quinoproteins, i.e. PQQ-containing enzymes (Duine \& Jongejan, 1989). The most-studied quinoproteins are methanol dehydrogenase from methylotrophs and glucose dehydrogenase (GDH, EC 1.1.99.17) from Gram-negative heterotrophic bacteria. The latter enzyme has been studied mainly in Acinetobacter calcoaceticus, which in fact contains two distinct GDHs: a soluble periplasmic one composed of two identical subunits of $48-54 \mathrm{kDa}$ (Dokter et al., 1986; Geiger \& Görisch, 1986) and a membrane-bound monomeric GDH of $83 \mathrm{kDa}$ (Matsushita et al., 1986, 1988; Cleton-Jansen et al., 1988).

GDH is widespread among the Enterobacteriaceae (Bouvet \& Grimont, 1988; Bouvet et al., 1989) but only some species such as Klebsiella aerogenes (K. pneumoniae) contain an active holoenzyme (Neijssel et al., 1983; Neijssel, 1987). Other species, including Escherichia coli, are seemingly unable to synthesize PQQ but they can

Abbreviations: PQQ, pyrroloquinoline quinone; GDH, glucose dehydrogenase; PTS, pts, phosphotransferase. produce the protein moiety, the apoenzyme, of GDH (Hommes et al., 1984). The apoGDH of E. coli has been purified and characterized by Ameyama et al. (1986): it is a membrane-bound protein of $88 \mathrm{kDa}$ which shows immunological relationships with the membrane-bound GDH of $A$. calcoaceticus and of Pseudomonas aeruginosa (Matsushita et al., 1986).

Reconstitution of the active holoenzyme of $E$. coli was obtained in vitro after incubation of PQQ with cell-free extracts (Hommes et al., 1984) or with membrane fraction or purified apoGDH (Ameyama et al., 1986). In vivo, holoenzyme reconstitution can be shown in $E$. coli PTS $^{-}$mutants. In these mutants the utilization of several carbohydrates, including glucose, is impaired by the deterioration of the phosphotransferase (PTS) system of concomitant phosphorylation and penetration of the carbohydrate substrate through the cytoplasmic membrane (reviewed by Postma et al., 1989; Erni, 1989). As a consequence, glucose catabolism in PTS $^{-}$mutants cannot proceed via the usual Embden-Meyerhof pathway. However, their growth on glucose can be restored by addition of PQQ to the culture medium (Hommes $e t$ al., 1984). In this situation, the reconstituted holoGDH oxidizes glucose into gluconic acid which is, in turn, presumably catabolized via the Entner-Doudoroff pathway (Gottschalk, 1985), thus providing an alternative route of glucose utilization.

Hommes et al. (1985) have shown that in K. aerogenes 
Table 1. Bacterial strains

\begin{tabular}{|c|c|c|}
\hline Strain & Characteristics & $\begin{array}{l}\text { Source or } \\
\text { reference }\end{array}$ \\
\hline \multicolumn{3}{|l|}{ E. coli } \\
\hline TP2111 & $\mathrm{F}^{-} x y l$ argH1 lacX74 aroB ilvA & Roy et al. (1983) \\
\hline TP2811 & Derived from TP2111; $\Delta(p t s H p t s I ~ c r r) \mathrm{Km}^{r}$ & Lévy et al. (1990) \\
\hline EF15 & A TP2811 derivative able to grow on glucose & This work \\
\hline FB8 & $F^{-} ;$wild-type & Bruni et al. (1977) \\
\hline FB8 $\Delta p t s$ & $\begin{array}{l}\text { A } \Delta(p t s H \text { ptsI crr }) \text { transductant from } \\
\text { FB } 8 \times \text { P1 TP2811 }\end{array}$ & This work \\
\hline CGSC 6902 & $\mathrm{~F}^{-}$his leu ilvA $\triangle$ lac mglP galP:: $\operatorname{Tn} 10$ & $\begin{array}{l}\text { B. J. Bachmann, E. coli Genetic } \\
\text { Stock Center, Yale University }\end{array}$ \\
\hline FB8 $\Delta p t s$ galP & $\begin{array}{l}\text { A galP: :Tn } 10 \text { transductant from } \\
\text { FB8 } \Delta p t s \times \text { P1 CGSC } 6902\end{array}$ & This work \\
\hline $\begin{array}{l}\text { EF16, EF17, EF18, } \\
\text { EF19, EF20 }\end{array}$ & FB8 $\Delta$ pts derivatives able to grow on glucose & This work \\
\hline $\begin{array}{l}\text { EF260, EF261, EF262, } \\
\text { EF263, EF264 }\end{array}$ & $\begin{array}{l}\text { FB8 } \Delta \text { pts galP derivatives able to grow on } \\
\text { glucose }\end{array}$ & This work \\
\hline \multicolumn{3}{|l|}{$\begin{array}{l}\text { Acinetobacter } \\
\text { calcoaceticus }\end{array}$} \\
\hline LMD79.42 & Wild-type & Dokter et al. (1986) \\
\hline
\end{tabular}

( $K$. pneumoniae), the oxidation of glucose by GDH is an auxiliary energy-generating pathway under conditions of energy stress, mainly phosphate or potassium limitation. This finding might perhaps be generalized to other PQQproducing members of the Enterobacteriaceae. However, for the species which apparently do not produce PQQ, the question arises whether they have the genetic complement required for PQQ biosynthesis. This question is not limited to $E$. coli and to Enterobacteriaceae containing an apoGDH. GDH has been found in many other bacterial genera, either as holoenzyme or as apoenzyme as in the Enterobacteriaceae. ApoGDH has been detected in species of Acinetobacter, Agrobacterium, Azotobacter, Rhizobium and Pseudomonas (Van Schie et al., 1985, 1987; Linton et al., 1987). Moreover, enzymes other than GDH may also be concerned; for example, Pseudomonas testosteroni contains a PQQ-dependent apoethanol dehydrogenase which is used for ethanol utilization only in the presence of added PQQ (Groen $e t$ al., 1986).

In this paper we describe the isolation and some characteristics of spontaneous mutants that arose from $E$. coli strains lacking $p t s$ genes, which recovered the ability to utilize glucose as growth substrate, with concomitant appearance of a functional holoGDH containing PQQ.

\section{Methods}

Media and growth conditions. The complex media were McConkey agar base (Difco), supplemented with a carbon source $(1 \%, w / v)$, and LB medium (Miller, 1972). The minimal medium was medium A (MA) of MacLennan et al. (1971) supplemented with vitamin B1 $\left(1 \mu \mathrm{g} \mathrm{ml}^{-1}\right)$, a filter-sterilized carbon source at $0.4 \%$ for liquid media or $1 \%$ for solid media and, if required, PQQ (Fluka) (1 nM) or amino acids (1 mM) to complement auxotrophic requirements. Antibiotics, when added, were used at a final concentration of $\left(\mu \mathrm{g} \mathrm{ml}^{-1}\right)$ : kanamycin, 25 ; tetracycline, 10. Solid minimal media contained Difco agar: $1.5 \%$ for Petri dishes or $0.6 \%$ for deep agar tubes ( $10 \mathrm{ml}$ medium in $16 / 160 \mathrm{~mm}$ tubes). Before use, the latter were degassed for $30 \mathrm{~min}$ in a boiling water-bath, equilibrated at $50^{\circ} \mathrm{C}$, evenly inoculated with $2-3 \times 10^{2}$ bacterial cells and immediately cooled to solidify the medium before incubation. Anaerobic cultures on the surface of agar plates were incubated in jars containing the GasPak (BBL) oxygen-absorbing mixture. Inocula for growth experiments in liquid minimal media were prepared from overnight cultures in LB medium, washed twice in MA medium without carbon source and adequately diluted. All the liquid cultures were strongly shaken with reciprocal shakers. Carbon source utilization tests (auxanograms) were done in the Unite des Entérobactéries, Institut Pasteur, by using experimental API strips which contained pure carbon sources inoculated with a bacterial suspension in a mineral base (Grimont et al., 1988). One hundred distinct carbon sources were tested. All the cultures were incubated at $37^{\circ} \mathrm{C}$.

Strains. These are listed in Table 1. E. coli TP2111 and FB8 had the typical substrate utilization profile of $E$. coli strains as recognized by API test auxanograms using defined culture media (Grimont $e t$ al., 1988). Using the same technique the PTS phenotype of $E$. coli TP2811 and of FB8 medium containing glucose, fructose, trehalose, mannose, mannitol, sorbitol, $N$-acetylglucosamine or methyl $\alpha$-D-glucopyranoside.

A phage P1 lysate obtained with TP2811 was used to transduce the pts deletion into FB8: on McConkey-glucose plates, the transductants FB8 $\Delta p t s$ gave white colonies. To obtain FB8 4 ts galP, a P1 lysate from $E$. coli CGSC 6902 was used to transduce FB8 $\Delta$ pts. The tetracyclineresistant colonies obtained were checked for their inability to grow on galactose MA medium.

Biochemical techniques. The different steps of preparation and solubilization of the membrane fraction containing GDH were essentially those of Ameyama et al. (1986) with minor modifications. Bacterial cells were suspended in Tris/ $\mathrm{HCl}$ buffer $(50 \mathrm{mM}, \mathrm{pH} 7)$ containing $\mathrm{MgSO}_{4},(2.5 \mathrm{mM})$ and passed twice through a French pressure cell at $110 \mathrm{MPa}$. Broken cell debris was removed by 
centrifugation at $6000 \mathrm{~g}$ for $10 \mathrm{~min}$ and the supernatant (crude extract) was ultracentrifuged at $80000 \mathrm{~g}$ for $90 \mathrm{~min}$. The resulting pellet was suspended in $1-2 \mathrm{ml}$ potassium phosphate buffer $(5 \mathrm{mM}, \mathrm{pH} 7)$ containing $\mathrm{MgSO}_{4}(2.5 \mathrm{mM})$ and Triton X-100 (1\%). This suspension was stirred for $2 \mathrm{~h}$ at $4{ }^{\circ} \mathrm{C}$, centrifuged at $80000 \mathrm{~g}$ for $90 \mathrm{~min}$ and the supernatant containing the solubilized membrane fraction was used for GDH assay and PQQ determination. GDH was assayed by the method of Ameyama et al. (1986) either without added PQQ to determine holoenzyme activity or with PQQ at a saturating concentration $(45 \mu \mathrm{M})$ for apoenzyme determination. Specific activity was expressed as $\mu \mathrm{mol}$ dichlorophenol indophenol reduced $\mathrm{min}^{-1}$ (mg protein) $)^{-1}$.

For PQQ determinations, samples of membrane preparation containing active GDH were heated for $5 \mathrm{~min}$ in a boiling water-bath (Biville et al., 1988). Free PQQ was assayed by a method using the apoethanol dehydrogenase purified from Pseudomonas testosteroni according to Groen et al. (1986). The reconstituted ethanol dehydrogenase activity was proportional to the amount of PQQ added from $0 \cdot 1$ to $5 \mathrm{pmol}$ in a total volume of $1 \mathrm{ml}$. All the GDH determinations and PQQ assays were achieved in triplicate with each membrane preparation.

Gluconic acid was determined with enzyme kits (Boehringer 428191) containing gluconate kinase and a NADP-dependent 6-phosphogluconate dehydrogenase. Production of gluconic acid by membrane preparations containing active GDH was achieved in a spectrophotometric cuvette containing, in a total volume of $1 \mathrm{ml}$ : membrane preparation $\left(50 \mu \mathrm{g}\right.$ protein); Tris $/ \mathrm{HCl}, 50 \mathrm{~mm} ; \mathrm{MgSO}_{4}, 2.5 \mathrm{~mm}$; glucose, $20 \mathrm{~mm}$; and Würster's Blue, $10 \mu \mathrm{M}$, as the primary electron acceptor. Potassium ferricyanide, the final electron acceptor, was added as $2 \mu \mathrm{l}$ fractions of a $10 \mathrm{~mm}$ solution in water. Complete reduction of each ferricyanide fraction was followed at $420 \mathrm{~nm}$ before addition of the next fraction. The system of electron acceptors PQQWürster's Blue-ferricyanide was that used by Frank et al. (1989). To check that potassium ferrocyanide, accumulated during gluconic acid production in the system described above, did not interfere with gluconic acid determination, the membrane preparation was replaced by a sample of crude extract from Acinetobacter calcoaceticus and glucose was replaced by arabinose. A. calcoaceticus extracts contain a high level of GDH (Dokter et al., 1986) which can oxidize several aldoses including arabinose (Goosen et al., 1987). Accumulation of potassium ferrocyanide to a concentration of $1.5 \mathrm{~mm}$ did not alter the blank controls of the gluconate assay system, nor the determination of known amounts of added gluconate.

\section{Results}

\section{Growth kinetics of E. coli pts mutants in glucose minimal medium}

The initial observation was a residual growth of $E$. coli PTS $^{-}$strains on glucose MA plates. The strains used were TP2811 and FB8 8 pts, both of which are deleted of $p t s H, p t s I$ and $c r r$, three genes coding for essential enzymes of the PTS system, Enzyme I, HPr and Enzyme IIIGlc. To examine this observation more thoroughly, the growth of an inoculum of $10^{5}$ bacteria $\mathrm{ml}^{-1}$ in glucose MA medium was followed by plate counts on LB medium. The growth curve obtained (Fig. 1, curve A) showed a first phase of slow growth (doubling time, $d=10-12 \mathrm{~h}$ ) lasting about $3 \mathrm{~d}$, followed by a phase of rapid growth $(d \simeq 1.4 \mathrm{~h})$. Samples taken at the end of the latter phase and plated on glucose MA medium gave rise

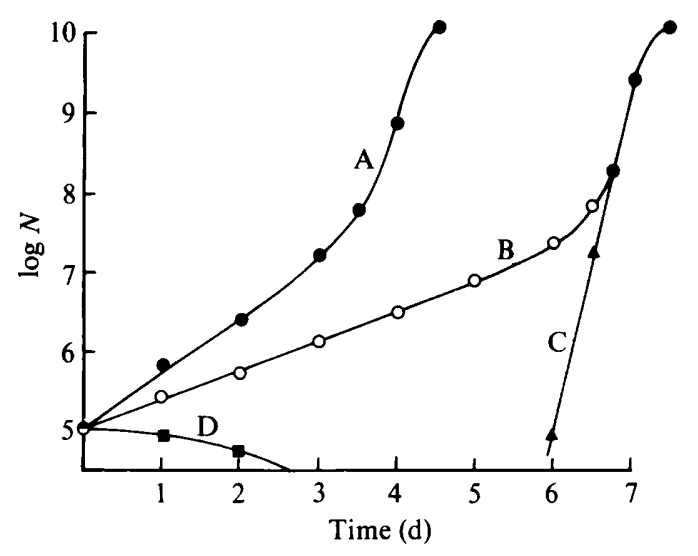

Fig. 1. Growth kinetics of $E$. coli FB8 $\Delta$ ts (curve A) and of FB8 $\Delta$ ts galP (curve B) in glucose MA medium. The population size, $N$ (c.f.u. $\mathrm{ml}^{-1}$ ), of curves A and $B$ was estimated by plating samples on LB medium. The differential count of EF mutants (curve C) emerging from the population of FB8 $\Delta$ pts galP was achieved on glucose minimal plates. The differential count of EF mutants obtained from FB8 $\Delta p t s$ is not represented (see text). Curve D: $E$. coli FB8 $\Delta p t s$ in minimal medium without glucose.

to colonies growing overnight in numbers comparable to those obtained on LB plates. From such glucose MA plates, strain EF15 was isolated from a culture of TP2811, and strains EF16 to EF20 were isolated from separate growth experiments with FB8 $\Delta$ ts. All the experiments reproduced the growth curve A of Fig. 1. The phenotypes of EF strains and of their respective parental strains TP2811 or FB8Dpts studied with API test auxanograms differed only in the ability to utilize glucose.

Saier et al. (1973), using a strain of Salmonella typhimurium deleted of essential phosphotransferase genes like our $E$. coli PTS $^{-}$strains, also obtained mutants growing rapidly in glucose minimal medium. These mutants were found to constitutively synthesize galactose permease. The broad specificity of this permease allowed glucose and some other PTS substrates to cross the cytoplasmic membrane. It was assumed that, after further phosphorylation by hexokinase, glucose was catabolized by the Embden-Meyerhof pathway. To check the possibility that our EF mutants were galactose permease constitutive mutants, the wild-type galP gene of FB8 $\Delta$ ts was replaced, by $\mathrm{P} 1$ transduction, by a galP gene inactivated by a $\operatorname{Tn} 10$ insertion. The resulting strain, FB8 $\Delta$ pts galP, was used for growth experiments in liquid MA medium as described above. Fig. 1 shows the similar shapes of the growth curves of the cultures of FB8 $\Delta$ pts (curve A) and of FB8 $\Delta$ pts galP (curve B). The growth of the latter, however, was slower during the first growth phase $(d=20 \mathrm{~h})$. As observed with FB8 $\Delta p t s$, cells of FB8 $\Delta$ pts galP growing rapidly in glucose MA medium 
Table 2. GDH activities and $P Q Q$ concentrations in membrane preparations of E. coli strains FB8 (wild-type), FB8 $\Delta$ ts and EF mutants

\begin{tabular}{|c|c|c|c|c|}
\hline \multirow[b]{2}{*}{ Strain } & \multirow{2}{*}{$\begin{array}{l}\text { Growth } \\
\text { conditions }\end{array}$} & \multicolumn{2}{|c|}{ GDH activity* } & \multirow{2}{*}{$\begin{array}{l}\text { PQQ } \\
\text { concn } \dagger\end{array}$} \\
\hline & & $-\mathrm{PQQ}$ & $+\mathrm{PQQ}$ & \\
\hline FB8 (w.t.) & Glucose & $<0.02$ & $0 \cdot 2-0 \cdot 4$ & $<0.5$ \\
\hline FB8 $\Delta p t s$ & $\begin{array}{l}\text { Glucose + } 1 \text { nM-PQQ } \\
\text { Glucose + } 100 \text { nM-PQQ }\end{array}$ & $\begin{array}{c}0 \cdot 1-0 \cdot 3 \\
1 \cdot 2\end{array}$ & $\begin{array}{c}0.2-0 \cdot 6 \\
1.3\end{array}$ & $\begin{array}{c}5-10 \\
30\end{array}$ \\
\hline EF $16,17,18,19,20$ & Glucose & $0 \cdot 1-0 \cdot 3$ & $0 \cdot 2-0 \cdot 6$ & $5-15$ \\
\hline $\begin{array}{l}\text { EF 260, 261, 262, } \\
263,264\end{array}$ & Glucose & $0 \cdot 1-0.3$ & $0.2-0.6$ & $5-15$ \\
\hline
\end{tabular}

*Units: $\mu \mathrm{mol}$ dichlorophenol indophenol reduced $\mathrm{min}^{-1}$ (mg protein $)^{-1}$.

$\dagger$ Units: pmol PQQ (mg protein) ${ }^{-1}$.

took over the whole culture during the second growth phase and they could be easily counted by plating on glucose MA medium, on which they produced colonies overnight. From separate growth experiments with FB8 8 ts galP, strains EF260 to EF264, growing rapidly in glucose MA medium, were isolated and their identity was checked as for strains EF16 to EF20.

From several experiments with FB8 $\Delta$ pts galP growing in liquid MA medium, it could be deduced, by extrapolation to $\log 0$ (i.e. one cell) of the straight line representing the EF cell population (Fig. 1), that the first EF cell appeared when the population reached approximately $5 \times 10^{6}-2 \times 10^{7}$ cells $\mathrm{ml}^{-1}$.

Direct plating of $2-4 \times 10^{8}$ cells of FB8 $\Delta$ pts onto glucose MA medium produced a heavy background of 'residual' growth over which colonies of rapidly growing EF cells could not be easily distinguished. Under the same experimental conditions, cells of FB8 $\Delta$ pts galP produced practically no background and isolated colonies of EF cells were fully developed in $48 \mathrm{~h}$. The frequency of appearance of EF cells, $2 \times 10^{-7}-5 \times 10^{-8}$, which was confirmed in this way, suggested that a single mutational event was involved in the appearance of EF cells in a pts population of $E$. coli.

\section{GDH determination and $P Q Q$ assays in membrane preparations}

Since the EF mutants were not galactose permease constitutive mutants, their selection under our experimental conditions could have resulted from the emergence of PQQ-producing cells in the PTS $^{-}$population, making possible the oxidation of glucose by a reconstituted GDH.

GDH activities in crude extract were too low to be correctly assayed, although non-specific dye reduction (i.e. before addition of glucose to the spectrophotometric cuvette) was very low. Supernatants after ultracentrifu- gation did not show any GDH activity: it was found only in the pellets solubilized by Triton X-100 treatment. Table 2 reports the results of apo- and holo-GDH activities and of PQQ assays in membrane preparations of $E$. coli FB8 (wild-type), FB8 $\Delta p t s$ and EF mutants. As expected, the membrane preparations of $E$. coli FB8 contained apo-GDH. Its specific activity was comparable to that found by Ameyama et al. (1986). Both holoGDH activity and PQQ concentration were below the limit of detection of the methods used.

The growth of FB8 $8 p t s$ in glucose MA medium required $\mathrm{PQQ}$ at the minimal concentration of $1 \mathrm{nM}$ to yield a development similar to EF strains both in growth rate and in final optical density. In these conditions, about half of the GDH was in the apo form. Addition of higher PQQ concentrations to the culture medium did not modify the growth parameters but GDH activity was increased and there was no free apoenzyme in the membrane preparation. An excess of PQQ, saturating all the available apoenzyme, perhaps had a stabilizing effect, explaining the larger amount of GDH in these preparations.

GDH activities and PQQ contents in the different preparations of all the EF mutants were found to be similar, as shown in Table 2. Comparison of GDH assays and $\mathrm{PQQ}$ concentrations in the different strains reported in Table 2 shows clearly that glucose utilization by EF strains was related to a synthesis of PQQ by these strains.

EF mutants derived from $E$. coli TP2811, such as EF15, could not be used for biochemical studies because the apoGDH was not stable during the procedure of membrane solubilization with Triton $\mathrm{X}-100$.

\section{Gluconic acid production by EF mutants}

Confirmation of the identity of the product of glucose oxidation by membrane preparations as gluconic acid was given by the coupled specificity of the two enzymes 


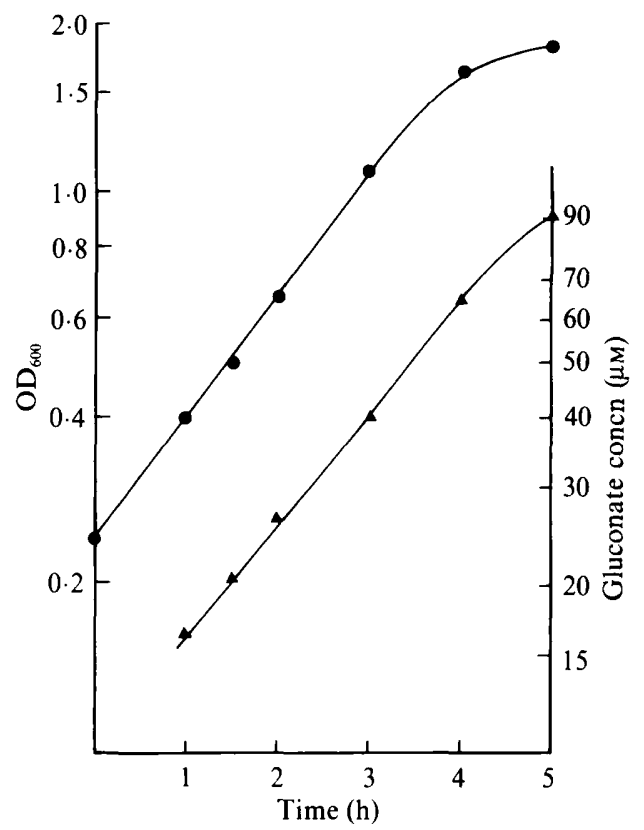

Fig. 2. Growth kinetics (O) of the $\mathrm{PQQ}^{+}$strain of $E$. coli $\mathrm{EF} 16$ in glucose MA medium and gluconate concentrations in the culture supernatant $(\mathbf{A})$.

used for the assay: gluconate kinase and NADPdependent 6-phosphogluconate dehydrogenase (Möllering \& Bergmeyer, 1984). No gluconic acid was produced in the presence of glucose 6-phosphate, indicating the absence of glucose-6-phosphate dehydrogenase in the samples used for assays.

In vitro, membrane preparations of EF cells grown in glucose MA medium catalysed stoichiometric oxidation of glucose into gluconic acid; in several experiments carried out as described in Methods, the reduction of $200 \mathrm{nmol}$ ferricyanide led to the production of $98 \mathrm{nmol}$ gluconic acid. Under the same experimental conditions, no gluconic acid was found when glucose was omitted from the production mixture nor when membranes without GDH activity were used.

In vivo, gluconic acid was detected in the supernatant of EF cells during the exponential phase of cultures in glucose MA medium (Fig. 2). The concentration of gluconic acid increased as the growth developed. Relative to the cell mass, this concentration remained practically constant $\left(40 \mu \mathrm{M}\right.$ per $\mathrm{OD}_{600}$ unit).

It was checked that in the supernatants of the overnight cultures in glycerol MA medium used as inoculum, no gluconic acid was detectable $(\leqslant 0.4 \mu \mathrm{M})$. The fact that gluconate did not accumulate in the culture medium suggests that it appeared as an intermediate of glucose catabolism rather than an end-product. In E. coli gluconate is catabolized by the Entner-Doudoroff pathway. Presumably, EF cells can dissimilate glucose by the Entner-Doudoroff pathway via gluconate formation, although we have no formal evidence for the existence of this catabolic route.

\section{Phenotype of EF mutants and relationships with oxygen}

All the EF strains isolated retained the whole phenotype of their parent strains except for the ability to utilize glucose in minimal medium. In the case of EF mutants obtained from FB8 $\Delta$ pts galP, a weak restoration of galactose utilization was observed, producing a slow and poor growth on galactose MA medium plates. This result is in accordance with the observation of Hommes $e t$ al. (1986), who described a PQQ-dependent galactose utilization by $E$. coli gal mutants.

In $E$. coli the reoxidation of reduced PQQ is linked to the respiratory chain (Van Schie et al., 1985; Matsushita et al., 1987). As a consequence, PTS $^{-}$strains in minimal medium supplemented with PQQ can grow only in the presence of oxygen. All the EF mutants utilized glucose only aerobically, as evidenced by the fact that in deep agar tubes containing glucose MA medium they grew only in the upper centimetre of the agar. Gluconate under the same conditions produced growth in the anaerobic as well as in the aerobic zone of deep agar tubes. These results demonstrated that only the step of glucose oxidation into gluconate was oxygen dependent, in accordance with the presence of a PQQ-dependent GDH in EF cells. Other non-PTS substrates tested allowed facultatively aerobic growth of EF strains as in the wild-type E. coli. All these results were confirmed in anaerobic jars.

The ability to use glucose only aerobically was used as a marker to check whether glucose-utilizing mutants other than EF cells were present in cultures of FB8 $\Delta p t s$ in experiments like that shown in Fig. 1. Two hundred colonies from LB count plates obtained from samples of different experiments taken at the beginning and at the end of the rapid growth phase were patched on glucose and gluconate MA media and incubated in air or in anaerobic jars. Patches of E. coli FB8 (wild-type) were added as a positive growth control on each plate. All the patches on gluconate plates grew with or without air, whereas on glucose plates all grew in the presence of air and none in anaerobiosis. This result showed that if glucose-utilizing mutants other than EF were present in this type of experiment, their proportion was always lower than $0.5 \%$.

\section{Discussion}

The EF mutants described in this work were $\mathrm{PTS}^{-} \mathrm{PQQ}^{+}$ strains of $E$. coli able to grow rapidly in glucose minimal 
medium. PQQ was synthesized in sufficient amount to promote a GDH specific activity similar to that found in $E$. coli $\Delta p t s$ grown in glucose minimal medium supplemented with $1 \mathrm{nM}-\mathrm{PQQ}$. The EF mutants could utilize glucose only aerobically, as expected from a PQQdependent glucose catabolism. The product of glucose oxidation by GDH, gluconate, was presumably catabolized via the Entner-Doudoroff pathway. These characteristics clearly distinguished EF mutants from the mutants of PTS ${ }^{-}$strains of Salmonella typhimurium described by Saier et al. (1973), which translocated glucose via a constitutive galactose permease. The fact that EF mutants could be obtained from $E$. coli $\Delta p t s$ galP eliminates the possibility of confusing the two types of mutants. Moreover, additional substrate utilization by EF mutants was limited to glucose whereas galactose permease constitutive mutants could grow with several PTS substrates (Saier et al., 1973). The reason why we did not find galPc mutants can only be a matter for speculation (difference of organisms, lower frequency of spontaneous mutation, mode of mutagenesis and of selection, etc.). The $S$. typhimurium mutants were selected after NTG mutagenesis, which was apparently without effect on the frequency of appearance of EF mutants in $E$. coli (unpublished results). Under our experimental conditions, spontaneous mutants producing measurable amounts of PQQ appeared at a frequency of $2 \times 10^{-7}-5 \times 10^{-8}$. They were selected by the strong selective pressure imposed on the population of $E$. coli $\Delta p t s$ cells by the glucose minimal medium.

Recently six $p q q$ genes involved in PQQ biosynthesis were cloned from Methylobacterium organophilum (Biville et al., 1989) and the cloned genes were used as hybridization probes in an attempt to identify similar genes in $E$. coli, but without success (F. Biville, unpublished results). Nevertheless, the experimental data presented here demonstrates that $E$. coli contains all the genetic information required for PQQ biosynthesis. If we consider EF mutants as regulatory mutants in which PQQ biosynthesis is derepressed, the target locus of the mutation was probably a genetic element involved in the level of expression of $p q q$ genes. We presently have no information on the nature of this locus. Attempts to alter a repressor gene by $\operatorname{Tn} 10$ chromosomal insertion in a pts strain did not yield EF strains (unpublished experiment).

Another possibility is the presence of a cryptic operon, expression of which might be triggered by an IS or Tn insertion upstream from $p q q$ genes. This type of regulation, which appears as the emergence of spontaneous mutations insensitive to chemical mutagens, has been described in several instances (for a review, see Discussion in Aronson et al., 1989).

Before any mutation affecting the expression of $p q q$ genes, the slow growth of $E$. coli $\mathrm{PTS}^{-}$in glucose minimal medium (Fig. 1) was caused by two distinct mechanisms. The difference in doubling time between the cultures of FB8 $\Delta p t s \quad(d=10 \mathrm{~h})$ and of FB8 $\Delta p t s$ galP $(d=20 \mathrm{~h})$ indicates a large participation of the galactose permease in the translocation of glucose through the cytoplasmic membrane, in accordance with the fact that about $10 \%$ of the fully induced permease activity was still present in uninduced cells, as was observed by Saier et al. (1973) for $S$. typhimurium. The nature of the growth observed after the galP inactivation is still unknown. Besides the possibility of an unknown system of glucose diffusion through the cytoplasmic membrane, a low level of PQQ production, not detectable by our assay method, is also possible; this could be demonstrated only with the use of a more sensitive means of PQQ detection.

The production of PQQ by E. coli was first claimed by Ameyama et al. (1984), who found a growth factor in culture supernatants which turned out to be PQQ. With our experimental conditions and assay method we did not confirm this result, since we never found any trace of PQQ ( $\leqslant 1 \mathrm{nM})$ in the culture supernatants. It is possible, however, that other strains or other experimental conditions might at least partially deregulate PQQ biosynthesis in E. coli.

In our work, PQQ was characterized by its activity as the apoenzyme of GDH from $E$. coli and of ethanol dehydrogenase from $P$. testosteroni used in the assay method. Duine et al. (1980) have shown that any modification of the PQQ molecule abolishes its activity as enzymic cofactor. This narrow specificity offered a good guarantee that the enzymic activities detected in this work were indeed caused by PQQ.

During the course of PQQ assays, we never experienced problems of unwanted PQQ in minimal culture media nor contaminant PQQ sticking to glassware as claimed by Van Kleef et al. (1987). The large number of our negative assays, the negative control and the everyday practice of $\mathrm{PQQ}$ assay in our laboratory attested the absence of contamination with correctly cleaned glassware. Moreover, the perfect reproducibility of the growth curves of FB8 $\Delta$ pts galP would have been impossible in contaminated vessels.

Klebsiella pneumoniae is an enteric species that produces PQQ. Recently Meulenberg et al. (1990) have shown that a PTS $^{-}$strain of this species can utilize glucose as growth substrate. This ability is lost in $\mathrm{PQQ}^{-}$ PTS $^{-}$mutants and restored after addition of PQQ to the culture medium. This situation is worth comparing with what we observed in E. coli. PQQ production in E. coli appeared only after a mutation allowing a $\mathrm{PTS}^{-}$strain to grow rapidly in glucose minimal medium. We have not yet selected $\mathrm{PQQ}^{-}$mutants of $E$. coli. In $E$. coli, as in $K$. pneumoniae, growth in glucose minimal medium of $p t s$ 
strains is associated with a PQQ-dependent oxidation of glucose into gluconate. How the gluconate resulting from this glucose oxidation is catabolized has not yet been analysed in $K$. pneumoniae or in $E$. coli. The most probable hypothesis is a dissimilation by the EntnerDoudoroff pathway as is the case for $E$. coli cultivated with gluconate as growth substrate (Gottschalk, 1985).

We thank Patrick Grimont and Elisabeth Ageron for performing auxanograms, Michael Herdman for critical reading of the manuscript, Sophie Lévy, Hilde de Reuse and Barbara Bachmann for providing strains, and J. Frank for the gift of Würster's Blue. This work was supported by grants from the Centre National de la Recherche Scientifique (URA1129) and from the EEC (ST2J-0478) to A. Danchin.

\section{References}

Ameyama, M., Shinagawa, E., Matsushita, K. \& Adachi, O. (1984). Growth stimulating substance for microorganisms produced by Escherichia coli causing the reduction of the lag phase in microbial growth and identity of the substance with pyrroloquinoline quinone. Agricultural and Biological Chemistry 48, 3099-3107.

Ameyama, M., Nonobe, M., Shinagawa, E., Matsushita, K., TAKImoto, K. \& ADACH, O. (1986). Purification and characterization of the quinoprotein D-glucose dehydrogenase apoenzyme from Escherichia coli. Agricultural and Biological Chemistry 50, 49-57.

aronson, B. D., Levinthal, M. \& Somerville, R. L. (1989). Activation of a cryptic pathway for threonine metabolism via specific IS3-mediated alteration of promoter structure in Escherichia coli. Journal of Bacteriology 171, 5503-5511.

Biville, F., Mazodier, P., Gasser, F., Van Kleef, M. A. G. \& Duine, J. A. (1988). Physiological properties of a pyrroloquinoline quinone mutant of Methylobacterium organophilum. FEMS Microbiology Letters 52, 53-58.

Biville, F., Turlin, E. \& Gasser, F. (1989). Cloning and genetic analysis of six pyrroloquinoline quinone biosynthesis genes in Methylobacterium organophilum DSM760. Journal of General Microbiology 135, 2917-2929.

Bouvet, O. M. M. \& GRIMONT, P. A. D. (1988). Extracellular oxidation of D-glucose by some members of the Enterobacteriaceae. Annales de l'Institut Pasteur/Microbiologie 139, 59-77.

Bouvet, O. M. M., Lenormand, P. \& Grimont, P. A. D. (1989). Taxonomic diversity of the D-glucose oxidation pathway in the Enterobacteriaceae. International Journal of Systematic Bacteriology 39, 61-67.

Bruni, C. B., Colantuoni, V., Sbordone, L., Cortese, R. \& Blasi, F. (1977). Biochemical and regulatory properties of Escherichia coli K12 hisT mutants. Journal of Bacteriology 130, 4-10.

Cleton-Jansen, A. M., Goosen, N., Wenzel, T. J. \& Van de Putte, P. (1988). Cloning of the gene encoding quinoprotein glucose dehydrogenase from Acinetobacter calcoaceticus: evidence for the presence of a second enzyme. Journal of Bacteriology 170, 2121-2125.

DokTer, P., FranK, J., JZn \& Duine, J. A. (1986). Purification and characterization of quinoprotein glucose dehydrogenase from Acinetobacter calcoaceticus LMD-79.41. Biochemical Journal 239, 163-167.

Duine, J. A. \& Jongejan, J. (1989). Quinoproteins, enzymes with pyrroloquinoline quinone as cofactor. Annual Review of Biochemistry 58, 403-426.

Duine, J. A. \& Jongejan, J. (1990). Pyrroloquinoline quinone: a novel cofactor. In: Vitamins and Hormones, vol. 45, pp. 223-262. Edited by G. D. Auerbach \& D. B. McCormick. London: Academic Press.

Duine, J. A., Frank, J., JZn \& Verwiel, P. E. (1980). Structure and activity of the prosthetic group of methanol dehydrogenase. European Journal of Biochemistry 108, 187-192.

ERNI, B. (1989). Glucose transport in Escherichia coli. FEMS Microbiology Reviews 63, 13-24.
Frank, J., Jr, VAN Krimpen, S. H., Van Verwiel, P. E. J., Jongejan, J. A., Mulder, A. C. \& DuINE, J. A. (1989). On the mechanism of inhibition of methanol dehydrogenase by cyclopropane-derived inhibitors. European Journal of Biochemistry 184, 187-195.

GEIGER, O. \& GöRISCH, H. (1986). Crystalline quinoprotein glucose dehydrogenase from Acinetobacter calcoaceticus. Biochemistry 25, 6043-6048.

Goosen, N., Vermaas, D. A. M. \& Van de Putte, P. (1987). Cloning of the genes involved in synthesis of coenzyme pyrroloquinolinequinone from Acinetobacter calcoaceticus. Journal of Bacteriology 169, 303-307.

GotTschalk, G. (1985). Bacterial Metabolism. The Entner-Doudoroff Pathway, 2nd edn., pp. 114-117. New York: Springer-Verlag.

Grimont, P. A. D., Jackson, T. A., Ageron, E. \& Noonan, M. J. (1988). Serratia entomophila, sp. nov., associated with Amber disease in the New-Zealand grass grub Costelytra zealandica. International Journal of Systematic Bacteriology 38, 1-6.

Groen, B., VAN Kleef, M. A. G. \& Duine, J. A. (1986). Quinohaemoprotein alcohol dehydrogenase apoenzyme from Pseudomonas testosteroni. Biochemical Journal 234, 611-615.

Hommes, R. W. J., Postma, P. W., Neijssel, O. M., Tempest, D. W., DoKTER, P. \& DuINE, J. A. (1984). Evidence of a quinoprotein glucose dehydrogenase apoenzyme in several strains of Escherichia coli. FEMS Microbiology Letters 24, 329-333.

Hommes, R. W. J., Van Hell, B., Postma, P. W., Neijssel, O. M. \& TEMPEST, D. W. (1985). The functional significance of glucose dehydrogenase in Klebsiella aerogenes. Archives of Microbiology 143, 163-168.

Hommes, R. W. J., Loenen, W. A. M., Neijssel, O. M. \& Postma, P. W. (1986). Galactose metabolism in gal mutants of Salmonella typhimurium and Escherichia coli. FEMS Microbiology Letters 36, $187-190$.

LÉvy, S., ZeNG, G.-Q. \& DANCHIN, A. (1990). Cyclic AMP synthesis in Escherichia coli strains bearing known deletions of the pts phosphotransferase operon. Gene 86, 27-33.

Linton, J. D., WoOdWARD, S. \& Gouldney, D. G. (1987). The consequence of stimulating glucose dehydrogenase activity by the addition of PQQ on metabolite production by Agrobacterium radiobacter NCIB1 1833. Applied Microbiology and Biotechnology 25, 357-361.

Maclennan, D. G., Ousby, J. C., Vasey, R. B. \& Cotton, N. T. (1971). The influence of dissolved oxygen in Pseudomonas AM1 grown on methanol in continuous culture. Journal of General Microbiology 69, 395-404.

Matsushita, K., Shinagawa, E., Inoue, T., Adachi, O. \& Ameyama, M. (1986). Immunological evidence of two types of PQQ-dependent $D$-glucose dehydrogenase in bacterial membranes and the location of the enzyme in Escherichia coli. FEMS Microbiology Letters 37, 141144.

Matsushita, K., Nonobe, M., Shinagawa, E., Adachi, O. \& AMEYAMA, M. (1987). Reconstitution of a pyrroloquinoline quinonedependent D-glucose oxidase respiratory chain of Escherichia coli with cytochrome $o$ oxidase. Journal of Bacteriology 169, 205-209.

Matsushita, K., Shinagawa, E., Adachi, O. \& Ameyama, M. (1988). Quinoprotein D-glucose dehydrogenase in Acinetobacter calcoaceticus LMD 79-41: the membrane-bound enzyme is distinct from the soluble enzyme. FEMS Microbiology Letters 55, 53-58.

Meulenberg, J. J. M., Sellink, E., Loenen, W. A. M., Riegman, N. H., Van KleEF, M. \& Postma, P. W. (1990). Cloning of Klebsiella pneumoniae $p q q$ genes and $\mathrm{PQQ}$ biosynthesis in Escherichia coli. FEMS Microbiology Letters 71, 337-344.

MiLLER, J. H. (1972). Experiments in Molecular Genetics, p. 433. Cold Spring Harbor, NY: Cold Spring Harbor Laboratory.

MöLleRING, H. \& BERGMEYeR, H. U. (1984). Methods of Enzymatic Analysis, vol. VI, pp. 220-227. Edited by H. U. Bergmeyer. Weinheim: Verlag Chemie.

NeIJssel, O. M. (1987). PQQ-linked enzymes in enteric bacteria. Microbiological Sciences 4, 87-90.

Neijssel, O. M., Tempest, D. W., Postma, P. W., Duine, J. A. \& FRANK, J., JZN (1983). Glucose metabolism by $\mathrm{K}^{+}$-limited Klebsiella aerogenes: evidence for the involvement of a quinoprotein glucose dehydrogenase. FEMS Microbiology Letters 20, 35-39. 
Postma, P. W., Broekruizen, C. P. \& Geerse, R. H. (1989). The role of PEP : carbohydrate phosphotransferase system in the regulation of bacterial metabolism. FEMS Microbiology Reviews 63, 69-80.

Roy, A., Haziza, G. \& Danchin, A. (1983). Regulation of adenylate cyclase synthesis in Escherichia coli: nucleotide sequence of the control region. EMBO Journal 2, 791-797.

Saier, M. H., Bramburg, F. G. \& Roseman, S. (1973). Characterization of constitutive galactose permease mutants in Salmonella typhimurium. Journal of Bacteriology 111, 512-514.

Van Kleef, M. A. G., Dokter, P., Mulder, A. C. \& Duine, J. A. (1987). Detection of cofactor pyrroloquinoline quinone. Analytical Biochemistry 162, 143-149.
Van Schie, B. J., Hellingwerf, K. J., Van Dijken, J. P., Elferink, M. G. L., VAN DiJl, J. M., Kuenen, J. G. \& Konings, W. L. (1985). Energy transduction by electron transfer via a pyrrolo-quinoline quinone dependent glucose dehydrogenase in Escherichia coli, Pseudomonas aeruginosa and Acinetobacter calcoaceticus (var. Iwoffi). Journal of Bacteriology 163, 493-499.

Van Schie, B. J., De Mooy, O. H., Linton, J. D., Van Dijken, J. P. \& KUENEN, J. G. (1987). PQQ-dependent production of gluconic acid by Acinetobacter, Agrobacterium and Rhizobium species. Journal of General Microbiology 133, 867-875. 